\title{
Correction
}

\section{Computer Imaging of the Atrioventricular Node and Bundles}

\author{
NK Roberts, KR Castleman
}

Ped Cardiol 1:275-279, 1980

Fig. 1 should have appeared as follows:
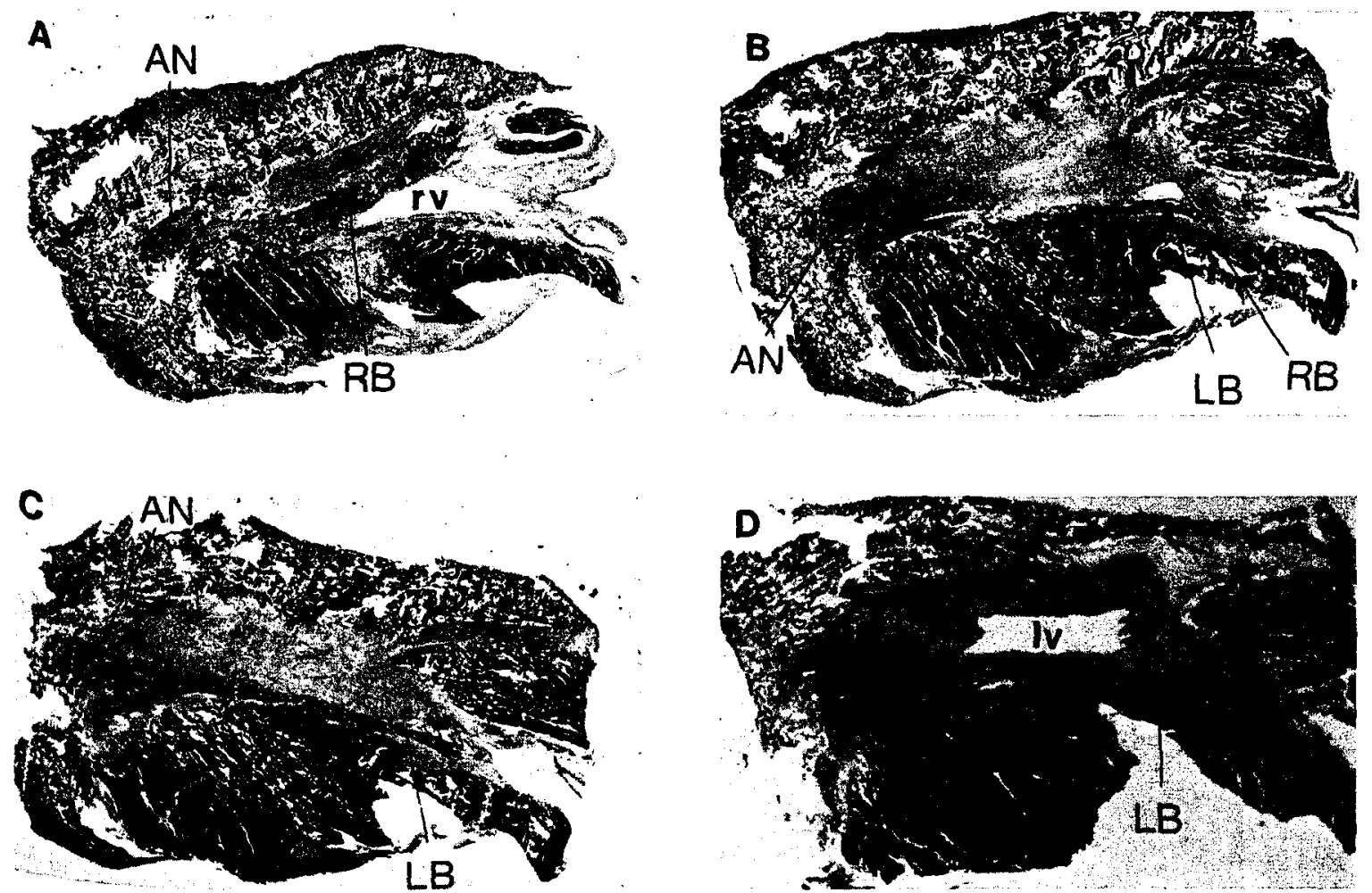

Fig. 1. S- $\mu \mathrm{m}$ serial sections of the atrioventricular node stained with hematoxylin, phloxine, and tartrozine (original magnification $\times 6$ ). A, The atrial muscle $(a t)$ surrounds the atrioventricular node $(A N)$; the right bundle $(R B)$ lies above the right ventricular cavity $(R V)$. See text for explanation of the arrow. B, Note the AN, the diminishing RB, and the appearance of the left bundle ( $L B$ ). The RB gets progressively smaller. $C$, The AN and atrioventricular bundle. D, The left ventricular cavity $(L V)$ increasing in size, and below it the LB. 

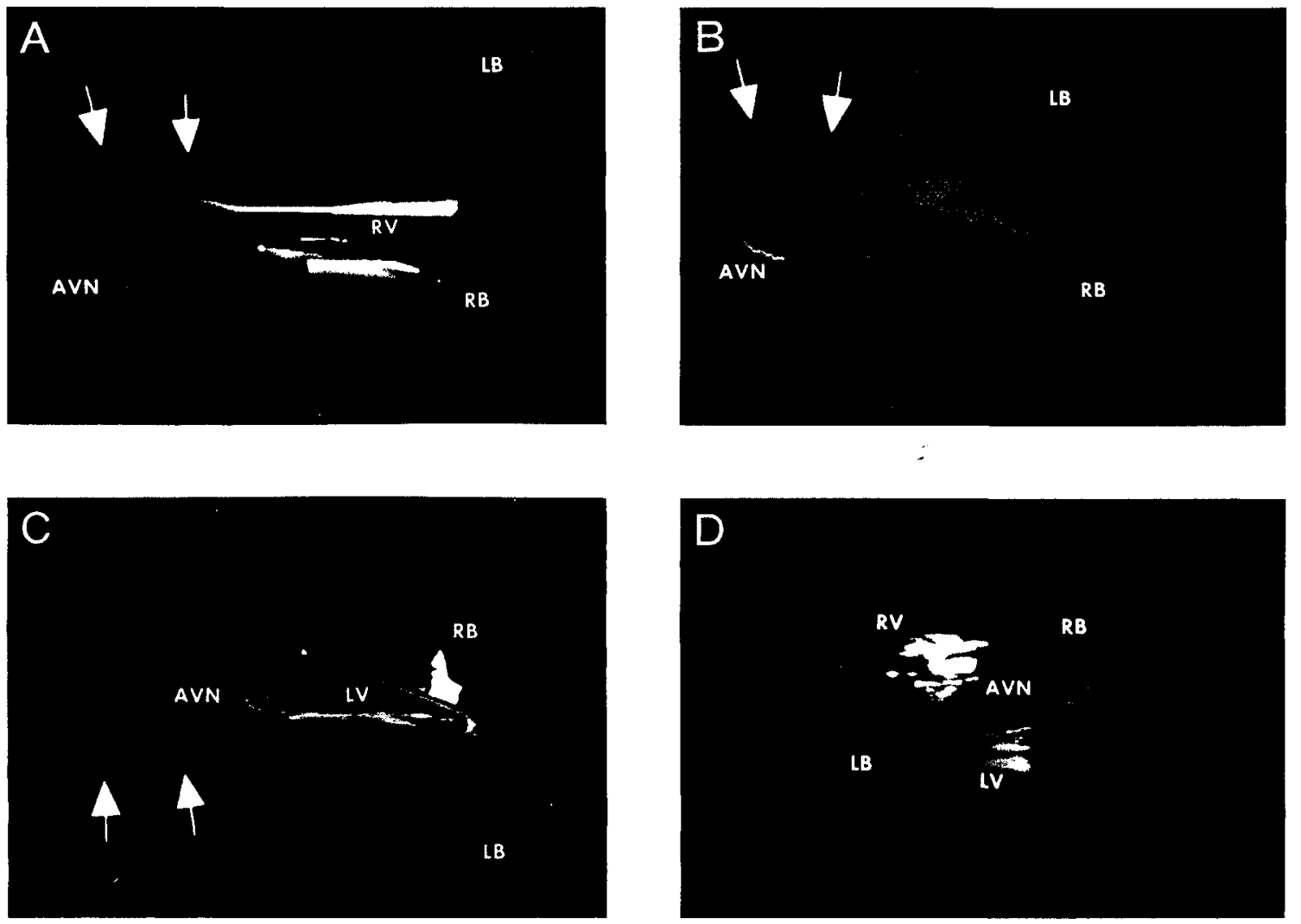

Fig. 3. Shaded-surface displays demonstrating the atrioventricular node (AVN), left bundle (LB), and right bundle (RB) in red, the right ventricular cavity $(R V)$ in green, and the left ventricular cavity $(L V)$ in blue. A and B show the same projection of display, but B has had the RV removed. These two figures are equivalent to Fig. $2 A$. In $C$, the structure is viewed from the left. In $D$, the AVN is viewed from the proximal and along the long axis of the node and bundle. See text for explanation of the arrows.

Above is an updated version of Fig. 3, with arrows shown as noted in caption. 